\title{
Real-time Doorway Detection and Alignment Determination for Improved Trajectory Generation in Assistive Mobile Robotic Wheelchairs.
}

\author{
Michael Gillham, Gareth Howells, Sarah \\ Spurgeon, Stephen Kelly \\ School of Engineering and Digital Arts \\ University of Kent \\ Canterbury, England, UK
}

\author{
Matthew Pepper \\ East Kent Hospitals University Foundation \\ Trust \\ Canterbury, England, UK
}

\begin{abstract}
Powered wheelchair users may find operation in enclosed environments such as buildings difficult; a fundamental problem exists: wheelchairs are not much narrower than the doorway they wish to pass through. The ability to detect and pass through doorways represents a major current challenge for automated guided wheelchairs. We utilize a simple doorway pattern recognition technique for fast processing in a real-time system for robotic wheelchair users. We are able to show a $96 \%$ detection and identification of 5 individual doorways and an $86 \%$ recognition rate of 22 separate approach angles and translations. We conclude that pattern recognition using features obtained from simple constrained infrared ranging sensor data binning can be utilized for fast identification of doorways, and important coarse position and approach angle determination, suitable for real-time trajectory adjustment, representing a significant enhancement in this area.
\end{abstract}

Keywords; robotic wheelchair; doorway approach angle; pattern recognition; assistive system; real-time; door passing

\section{INTRODUCTION}

To date, research into assistive systems for wheelchair users has mainly considered the autonomous robotic approach where control is taken away from the user rather than providing any measured amount of assistance $[1,2]$.

In order to provide any seamless robotic-human assistive system, with varying levels of assistance, a sense of human like perception must be first considered [3]. One such assistive requirement might be offering corrective doorway passing trajectory feedback to users of powered wheelchairs, necessitating pre-emptive real-time doorway recognition and identification, from unknown and unpredicted user generated approaches, rather than autonomously planned pathways, which are executed much faster than an autonomous system can operate.

Most research in this area has considered autonomous assistance $[1,4]$ in public undefined locations. However there may well be attendants and assistance on hand to offer support. However, in practice the wheelchair user is likely to spend more time in familiar surroundings such as their home or work place, a situation they may well feel comfortable in and therefore overconfident in use of their wheelchairs. A significant problem exists, which makes doorway alignment critical, in that wheelchair width is not much narrower than standard doorways resulting in many collisions; a situation which may commonly be observed with manual wheelchairs, one which can inhibit use of the wheelchair for both user and carer. Therefore any assistive system must run real-time monitoring in the background ready to assist robustly, warning what corrective measures are required and what intervening action the system can take, if any, thus empowering users $[1,5]$.

The doorway passing wheelchair problem may be considered a 'peg-through-the-hole' problem, where depth of the hole provides information for alignment purposes [6]. Depth in this case is minimal and therefore alignment difficult, different algorithms and sensors may be used for general obstacle avoidance and user feedback than those, imposed harder constraints, required for doorway passing; thus doorway identification becomes a crucial issue.

Users may well not think they need assistance [7], thus early doorway detection offers real-time user trajectory correction feedback. This would improve orthogonal lineup and would thus maximize clearance between doorway and the wheelchair, leading to collision elimination. Additionally if required, users could initiate an automated doorway passing system if they felt in need of assistance.

Door openings have another component, the door, which makes detection difficult. In real situations however, most doors, when considering wheelchair users, either open automatically to a set point, or in the home, may be removed and, where not, would most likely be propped open when human assistance is unavailable. Therefore, utilizing a pattern-recognition trained system; various doorway configurations can be stored. The trained system can then be tested in real-time to answer the following questions:

- Is it a doorway or not?

- Which specific doorway is it?

- What trajectory corrections are required if any?

Answering these questions using current sensor readings and stored information allows generation of assistive trajectories, thus providing early user feedback; 
users may then self-correct before intervention becomes necessary. Furthermore, storage of intervention information by the system would give medical practitioners valuable information, otherwise requiring extensive and expensive medical testing, with regard to long term motor/cognitive deterioration in the user. Additionally, for any practical employable robust assistive solution to doorway passing the following criteria need to be met:

- No moving parts.

- Low cost.

- Simple processing.

- Human interaction compatible.

Pattern recognition systems can be easily trained in the field with unique locations and users; re-training if conditions change, such as door prop angles, or furniture location change, or clutter build-up, particularly in the case of sensor wear and mechanical misalignment. We therefore propose a method of doorway detection and recognition based upon pattern recognition in a dynamic human environment for the purpose of trajectory adjustment in human assistive robotic platforms.

\section{SENSOR CHOICE AND METHODOLOGY}

A suitable sensor arrangement must be identified for doorway identification. Feature identification using geometric ranging information has been covered previously in the literature [8-11]. Sonar has been used to detect corners and junctions [12], and doorways [9] although in this case detection was based upon wall following/corridor methods as the robot passed the doorway rather than approaching from deep inside rooms.

Other sensors employed, such as the scanning laser, have succeeded [4] in locating doorways geometrically and generating trajectories. However laser scanning sensors require time to scan and are currently expensive and bulky. The infrared Sharp GP2Y0A710K0F ranging sensor is inexpensive and has a maximum $5 \mathrm{~m}$ range; these types of sensors have been used previously for statistical pattern recognition [11] and detecting corners, junctions and corridor doorways [13].

\section{FEATURES AND CLASSIFICATION}

In order to provide a fast response system, after initial experimentation, we chose to use binning from the ranging sensors as our features set. Further, we chose to use high resolution raw data $(\mathrm{mm})$ initially, and then lower resolution raw data $(\mathrm{cm})$ and finally a constrained binning where we simply take the difference between the highest range value and others to give an effective door shape dependent on platform rotation and translation with respect to the doorway. 1-nearest-neighbor (K-NN), Bayes-Normal-1, Bayes-Normal-2 and Naive Bayes classifiers were employed to test the doorway and approach position identification.

Infrared analog ranging sensors have been used successfully for statistical object identification (100\%) [11] and accurate mapping [14]. Similar pattern recognition work undertaken on embedded processing time for various online classifiers [15] indicates K-NN took 200 times longer than Bayes-Normal-2 which was capable of running similar pattern recognition tasks in the microsecond range on a dual core $1500 \mathrm{MHz} 2 \mathrm{~GB}$ RAM MICROSPACE EBX (MSEBX945) small computer format board with 2000 sample training set and 100 features. Other work used weightless neural networks for simple geometric pattern recognition, similar to our work presented in this paper, with an execution time of $0.25 \mu \mathrm{s}$ on an Atmel AT89x55 24.3MHz processor with 256 bytes of RAM [12]. We show in our results that Bayes-Normal1 and 2 classifiers can produce acceptable results which are suitably fast for real-time online application [15] particularly with such reduced feature set.

\section{RESULTS}

A room with multiple doorway configurations and a flat suitable for wheelchair user occupation together with several other typical office-like-rooms were tested for doorway profile and approach angle identification. Data was collected in real-time doorway approaches and rooms were thoroughly scanned for comparison. Re-testing was randomly undertaken at a later time confirming the results were consistent and true.

A standard doorway in a large cluttered room with three possible door openings was initially tested. Seven approach angles were used $[-45,-30,-15,0,15,30,45$ degrees] with respect to the doorway midpoint (2600 samples average) and the room extensively sampled $(>18000$ ) the data being split 60:40 (other ratios were examined without significant change) for training and testing respectively; ranging data was smoothed with $\mathrm{mm}$ resolution and sampled $>200 / \mathrm{s}$. According to the results listed in Table. 1, the 1-NN classifier clearly outperforms the others with respect to identification of individual approach angles where consistency was lacking. 
TABLE I. INITIAL DOORWAY TESTING

\begin{tabular}{|c|c|c|c|c|}
\hline \multirow[b]{2}{*}{ Feature tested } & \multicolumn{4}{|c|}{ Percentage of correctly identified samples } \\
\hline & $\begin{array}{c}1-N N \\
\text { Classifier }\end{array}$ & $\begin{array}{c}\text { Bayes- } \\
\text { Normal-1 }\end{array}$ & $\begin{array}{c}\text { Bayes- } \\
\text { Normal-2 }\end{array}$ & $\begin{array}{l}\text { Naive } \\
\text { Bayes }\end{array}$ \\
\hline \multicolumn{5}{|c|}{ Left inward opening door } \\
\hline 7 approach angles & 93.2 & 54.6 & 76.3 & 66.4 \\
\hline Non-doorway & 93.6 & 63.7 & 79.4 & 58.5 \\
\hline \multicolumn{5}{|c|}{ Opening only no door } \\
\hline 7 approach angles & 92.4 & 58.9 & 49.3 & 67.9 \\
\hline Non-doorway & 94.0 & 67.4 & 47.0 & 66.4 \\
\hline \multicolumn{5}{|c|}{ Right inward opening door } \\
\hline 7 approach angles & 94.9 & 70.8 & 58.4 & 83.8 \\
\hline Non-doorway & 96.4 & 73.8 & 40.4 & 78.9 \\
\hline \multicolumn{5}{|c|}{ All three door openings } \\
\hline $\begin{array}{l}\text { Identify all } 21 \\
\text { individual } \\
\text { approach angles }\end{array}$ & 87.0 & 39.8 & 26.7 & 46.9 \\
\hline $\begin{array}{l}\text { Identify all } 21 \\
\text { approach angles } \\
\text { as doorway class } \\
\text { v non-doorway }\end{array}$ & 96.8 & 80.5 & 88.8 & 78.3 \\
\hline
\end{tabular}

When we combined all three door openings, same doorway same room, the confusion matrix, shown as a bitmap in Fig. 1, right, clearly indicates confusion between the three door openings, thus implying the free space/surroundings shape is a more prominently distinctive feature than the door its self.

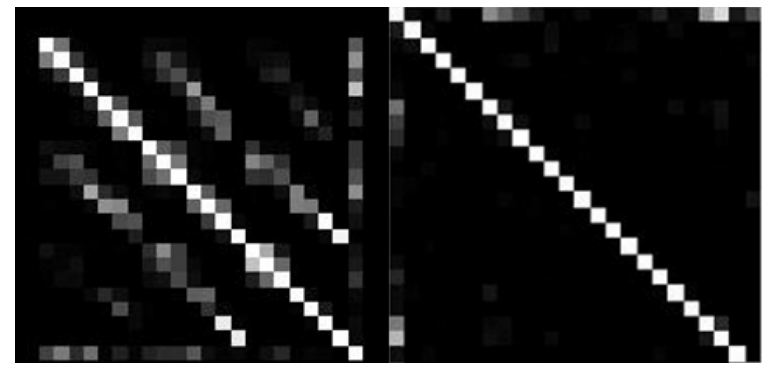

Fig. 1. 1-NN Classifier Confusion Bitmap Matrices, left image: initial doorway testing, and right image: various doorways and approach angles. Axes are true label top left to bottom left (ordered) and determined label top left to top right, white intensity represents sample count identified proportionally.

Confusion with the room is minimal and indeed all classifiers performed well in this respect; the Bayesian classifiers, all door orientations combined, show significant improvement over individual door positions, re-enforcing the door opening or hole as the prominent feature, where poor statistical performance indicates a required feature separation improvement.

We revised the feature range histogram by taking the maximum range from all the sensors and subtracting their values from that to give a distinctive shape representative of the geometric door and opening (1).

$$
\bar{K}=K_{\max }-K_{i} \text { where }\left\{\begin{array}{l}
i=1,2,3 \ldots n \\
K_{\max }=\max <K_{i}
\end{array}\right\}
$$

We tested this new pattern with a standard door opening, one correct angular and 3 correct translation approaches, and 8 other angular incorrect approaches together with 10 incorrect translations all within a $2.5 \mathrm{~m}$ range from and $2 \mathrm{~m}$ width zone immediately in front of the door opening. Angular range was -35 to $-15^{\circ},-15^{\circ}$ to $-5^{\circ}$, $5^{\circ}$ to $5^{\circ}, 5^{\circ}$ to $15^{\circ}$ and $15^{\circ}$ to $35^{\circ}$ offset such that each translation and the offset angles occupied a geometric area $30 \mathrm{~cm}$ by $40 \mathrm{~cm}$ each of the other angles represented a slice segmented every $40 \mathrm{~cm}$.

TABLE II. DOORWAY APPROACH ANGLE AND DISPLACEMENT

\begin{tabular}{|l|c|c|c|c|}
\hline \multirow{2}{*}{ Feature tested } & \multicolumn{4}{|c|}{ Percentage of correctly identified samples } \\
\cline { 2 - 5 } & $\begin{array}{c}\text { I-NN } \\
\text { Classifier }\end{array}$ & $\begin{array}{c}\text { Bayes- } \\
\text { Normal-1 }\end{array}$ & $\begin{array}{c}\text { Bayes- } \\
\text { Normal-2 }\end{array}$ & $\begin{array}{c}\text { Naive } \\
\text { Bayes }\end{array}$ \\
\hline $\begin{array}{l}\text { Approach } \\
\text { positions/angles }\end{array}$ & 86.6 & 51.7 & 72.7 & 62.6 \\
\hline
\end{tabular}

All 22 positions and angles were tested against each other in a 60:40 training/testing split and 4 random retests at some other time consistently confirming correct labeling.

The results in Table. 2, show that each rotation and translation can be identified by $1-\mathrm{NN}$ at $87 \%$ and by Bayes-Normal-2 at $73 \%$ correctly, the confusion matrix importantly determined that all of the errors were adjacent zones. An average 3000 samples were used for training and testing for each of the 22 positions/angles.

TABLE III. VARIOUS DOORWAYS AND APPROACH ANGLES

\begin{tabular}{|l|c|c|c|c|}
\hline \multirow{2}{*}{ Feature tested } & \multicolumn{4}{|c|}{ Percentage of correctly identified samples } \\
\cline { 2 - 5 } & $\begin{array}{c}1-N N \\
\text { Classifier }\end{array}$ & $\begin{array}{c}\text { Bayes- } \\
\text { Normal-1 }\end{array}$ & $\begin{array}{c}\text { Bayes- } \\
\text { Normal-2 }\end{array}$ & $\begin{array}{c}\text { Naive } \\
\text { Bayes }\end{array}$ \\
\hline $\begin{array}{l}\text { Approach } \\
\text { positions/angles }\end{array}$ & 96.3 & 67.8 & 85.0 & 66.8 \\
\hline
\end{tabular}

Finally testing the method we drove the wheelchair around a series of six rooms and through each of the 6 doorways at 3-4 different angles. We were able to determine the unique identification of individual doorway trajectory when compared to each and all others and to all of the rooms. Very little confusion occurred, as shown in Fig.1, left. Occasionally some trajectory samples were 
misidentified as room although they tended to be those at longer range. An average 1000 samples for each approach angle and 6500 samples for each room giving a 62000 total sample size and the geometric pattern with 8 features were employed.

Previous work has commonly used histogram grid map comparison. One such work combined sonar, image and digital infrared (fixed distance threshold not analog as we have used) [8] identifying 24 features, compared to our 8 , from all three sensor grid maps, and they achieved a $98 \%$ door recognition rate up to $2 \mathrm{~m}$ into the room using a computationally more expensive $3-\mathrm{NN}$ classifier, which is similar to our result of $96 \%$ from a $1-\mathrm{NN}$ classifier. Other work when looking for door openings (the gap) rather than the profile [3], using laser scanning and Scale Invariant Feature Transform identifying objects from imagery, noted false positives from the doorway detection, although a $93 \%$ to $97 \%$ trajectory correct classification success rate, whilst not directly and intentionally assessing doorway approach trajectories as we have done. However they note the process is significantly slow and requires a 360 degree scanning laser and a camera adding complexity, cost, and vulnerability and, hence, being unsuitable for a real-time human interactive system.

\section{CONCLUSION}

We have presented in this paper an application of pattern recognition using simple low cost ranging sensors to the significant and unsolved task of doorway identification, approach angles and position for the purpose of integration into a real-time trajectory assistive system such that correction, if possible, can be determined and notified to the user.

Contributions have been in the field of sensor complexity and cost reduction, robustness by removal of the need for scanning using a simple feature obtained from real-time sensor data, removing the need for complex mapping. We have also contributed by identification of doorway approach position and angle using a classifier algorithm suitable for real-time processing giving an accuracy of $85 \%$, where errors are geometrically adjacent classes, and unique identification of doorways themselves.

It is hoped that incorporating this system into the powered chair will enhance the independence and quality of life of the user and their care network.

\section{ACKNOWLEDGEMENTS}

Research part financed by European Union ERDF (European Regional Development Fund) through the Interreg IVA "2 Mers Seas Zeeën" cross-border cooperation program (2007-2013).

\section{REFERENCES}

[1] P. Nisbet, "Who's intelligent? wheelchair, driver or both?" IEEE International Conference on Control Applications, Glasgow, Scotland, 2002.

[2] C. De La Cruz, W. C. Celeste and T. F. Bastos, "A robust navigation system for robotic wheelchairs," Control Eng. Pract., vol. 19, pp. 575590,2011

[3] H. Zender, O. Martínez Mozos, P. Jensfelt, G. Kruijff and W. Burgard, "Conceptual spatial representations for indoor mobile robots," Robotics and Autonomous Systems, vol. 56, pp. 493-502, 2008.

[4] F. Auat Cheein, C. De La Cruz, R. Carelli and T. F. Bastos-Filho, "Solution to a door crossing problem for an autonomous wheelchair," in Intelligent Robots and Systems. IEEE/RSJ International Conference on, 2009, pp. 4931-4936.

[5] T. Carlson and Y. Demiris, "Increasing robotic wheelchair safety with collaborative control: Evidence from secondary task experiments," in Robotics and Automation (ICRA). IEEE International Conference on, 2010, pp. 5582-5587.

[6] H. Bruyninckx, S. Dutre and J. De Schutter, "Peg-on-hole: A model based solution to peg and hole alignment," in Robotics and Automation. IEEE International Conference on, 1995, pp. 1919-1924.

[7] C. Urdiales, M. Fernandez-Carmona, J. M. Peula, R. Annicchiaricco, F. Sandoval and C. Caltagirone, "Efficiency based modulation for wheelchair driving collaborative control," in Robotics and Automation (ICRA), IEEE International Conference on, 2010, pp. 199-204.

[8] J. D. Courtney and A. K. Jain, "Mobile robot localization via classification of multisensor maps," in Robotics and Automation. IEEE International Conference on, 1994, pp. 1672-1678.

[9] L. Kleeman, "Scanned monocular sonar and the doorway problem," in Intelligent Robots and Systems' 96, IROS 96, Proceedings of the 1996 IEEE/RSJ International Conference on, 1996, pp. 96-103.

[10] P. Carinena, C. V. Regueiro, A. Otero, A. J. Bugarin and S. Barro, "Landmark detection in mobile robotics using fuzzy temporal rules," Fuzzy Systems, IEEE Transactions on, vol. 12, pp. 423-435, 2004.

[11] B. Barshan, T. Aytaç and Ç. Yüzbaşı lu, "Target differentiation with simple infrared sensors using statistical pattern recognition techniques," Pattern Recognit, vol. 40, pp. 2607-2620, 2007.

[12] S. Nurmaini, S. Zaiton, M. Hashim and D. Jawawi, "Modular Weightless Neural Network Architecture for Intelligent Navigation," Int. J. Advance. Soft Comput, vol. 1, 2009.

[13] M. Gillham, B. McElroy, G. Howells, S. Kelly, S. Spurgeon and M. Pepper, "Weightless neural system employing simple sensor data for efficient real-time round-corner, junction and doorway detection for autonomous system path planning in smart robotic assisted healthcare wheelchairs," in Emerging Security Technologies (EST). Third International Conference on, 2012, pp. 161-164.

[14] H. Kim, Y. Ha and G. Jin, "A study on the environmental map building for a mobile robot using infrared range-finder sensors," in Intelligent Robots and Systems, IROS 2003 Proceedings. IEEE/RSJ International Conference on, 2003, pp. 711-716.

[15] A. Starzacher and B. Rinner, "Evaluating KNN, LDA and QDA classification for embedded online feature fusion," in Intelligent Sensors, Sensor Networks and Information Processing, ISSNIP 2008. International Conference on, 2008, pp. 85-90. 